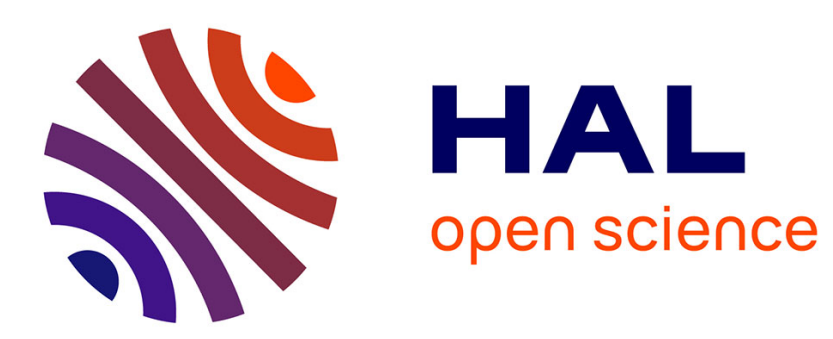

\title{
Multinomial model-based formulations of TCP and NTCP for radiotherapy treatment planning
}

\author{
Roukaya Keinj, Thierry Bastogne, Pierre Vallois
}

\section{To cite this version:}

Roukaya Keinj, Thierry Bastogne, Pierre Vallois. Multinomial model-based formulations of TCP and NTCP for radiotherapy treatment planning. Journal of Theoretical Biology, 2011, 279 (1), pp.55-62. 10.1016/j.jtbi.2011.03.025 . hal-00588935

\section{HAL Id: hal-00588935 \\ https://hal.science/hal-00588935}

Submitted on 27 Apr 2011

HAL is a multi-disciplinary open access archive for the deposit and dissemination of scientific research documents, whether they are published or not. The documents may come from teaching and research institutions in France or abroad, or from public or private research centers.
L'archive ouverte pluridisciplinaire HAL, est destinée au dépôt et à la diffusion de documents scientifiques de niveau recherche, publiés ou non, émanant des établissements d'enseignement et de recherche français ou étrangers, des laboratoires publics ou privés. 


\title{
Multinomial model-based formulations of TCP and NTCP for radiotherapy treatment planning
}

\author{
R. Keinj ${ }^{\text {a }}$, T. Bastogne ${ }^{\mathrm{b}, *}$, P. Vallois ${ }^{\mathrm{a}}$. \\ a INRIA-BIGS \& Institut de Mathématiques Elie Cartan, \\ Nancy-Université, CNRS UMR 7502, \\ BP 239, F-54506 Vandouvre-lès-Nancy Cedex, France \\ b INRIA-BIGS \& Centre de Recherche en Automatique de Nancy (CRAN) \\ Nancy - Université, CNRS UMR 7039, \\ BP 239, F-54506 Vandœuvre-lès-Nancy Cedex, France, \\ Phone: (33) 383684473 - Fax: (33) 383684462
}




\begin{abstract}
Hit and target models of tumour growth, typically assume that all surviving cells have a constant and homogeneous sensitivity during the radiotherapy period. In this study, we propose a new multinomial model based on a discrete-time Markov chain, able to take into account cell repair, cell damage heterogeneity and cell proliferation. The proposed model relies on the 'Hit paradigm' and 'Target' theory in radiobiology and assumes that a cancer cell contains $m$ targets which must be all deactivated to produce cell death. The surviving cell population is then split up into $m$ categories to introduce the variation of cancer cell radio-sensitivity according to their damage states. New expressions of the tumour control probability (TCP) and normal tissue complication probability (NTCP) are provided. Moreover, we show that hit and target models may be regarded as particular cases of the multinomial model. Numerical results should permit to keep the efficiency of treatment with a lower total radiation dose then that given by the typical hit models, which allows to decrease side effects.
\end{abstract}

Key words: Markov chain, multinomial model, tumor growth, radiotherapy

\footnotetext{
$\bar{*}$ Corresponding author.

Email address: thierry.bastogne@cran.uhp-nancy.fr (T. Bastogne).
} 


\section{Introduction}

The aim of radiotherapy is to deliver enough radiation to the tumour to control it without irradiating normal tissue to a dose that will lead to serious complications (morbidity). Since radiation delivery at a precise point of cells is generally described as a random variable, the effects of the radioactive treatments on cancer and healthy cells are characterized by two probabilities: (i) the Tumour Control Probability (TCP) and (ii) the Normal Tissue Complication Probability (NTCP $)^{3-5}$. The optimum choice of radiation dose delivery technique in the treatment of a given tumour has to maximize the TCP so that at the same time the NTCP must be lower than an acceptable level. The model-based probabilities TCP and NTCP are then used to choose the 'best' treatment plan. Since Holthusen in ${ }^{6}$, TCP and NTCP dose functions have been used to determine the 'optimal' dose to be delivered by maximizing biological objective functions like $T C P(1-N T C P)$. An evolution of this objective function is proposed in $^{7}$. In clinical radiotherapy a typical choice is $T C P \geq 0.5$ and $N T C P \leq 0.05^{8}$. Expressions for TCP and NTCP can be derived using survival curves ${ }^{9}$, stochastic cell population-dynamic models ${ }^{10,11}$ or cell-cycle models ${ }^{12}$. Here, we focus on a popular class of models used in radiotherapy, i.e. the hit models coming from target theory.

Target theory and hit-modeling paradigm were introduced in the 1920s when biologists were beginning to develop quantum approaches to inactivation phenomena in irradiated biological tissue ${ }^{13-15}$. The modeling of radiation effects on living cells were continued both theoretically and experimentally by K. C. Atwood and A. Norman ${ }^{16}$, D. E. Lea ${ }^{17}$, E. C. Pollard and coworkers ${ }^{18,19}$. Since these seminal works, a lot of mathematical models expressing the interaction 
of radiation particles with biological cells have been proposed ${ }^{20-25}$. In target theory, a cell has different vital sites called targets which must be inactivated to kill the cell. For instance, it is accepted that the chromosomes are sensitive targets $^{26}$ but there is additional evidence that the nuclear membrane, or some cell organelles close to the nuclear membrane can also be regarded as targets ${ }^{27}$. Each target is deactivated when it is hit by a number of radiation particules. There are several classes of hit models classified by the number of targets and the number of hits. In these models, it is generally assumed that cells have homogeneous behaviour. In practice, there are at least three main reasons to put this hypothesis into question. The first cause of heterogeneity comes from the nonuniform spatial distribution of the radiation dose. The second cause is due to the differences between the cell types (necrotic, quiescent, proliferating, stem cells, etc.) and the nonuniform concentration of oxygen and nutrients. A third factor corresponds to a cell-to-cell variability of damages and to the variation of the cell sensitivity to radiation. The first two points may be handled by reducing the modeling scale to a voxel level, i.e. a sub-volume in which we can reasonably suppose that distribution doses and cell types are homogeneous. For the last point, the hit models typically assume that cell sensitivity to radiation is constant over the time course of radiotherapy. In other words, after a radiation dose, a surviving cell is thought to be about as viable as an unirradiated cell and all cells are supposed having the same survival probability. However, evidence suggests that the cell radiation sensitivity increases with the number of dose fraction and even the cell is still alive, it partially loses the ability to resist as an unirradiated cell. Our aim is to focus on this last issue and a triple objective is addressed:

- to propose an extension of hit models based on a Markov chain formalism 
able to describe the target reparation between two consecutive dose fractions of the radiation schedule, the heterogeneity of damages induced by radiations and the cell proliferation;

- to determine the TCP and NTCP;

- through numerical computations, we compare the multinomial model with typical hit models and we draw the optimal total dose from the TCP/NTCP curves.

This paper is structured as follows: we present a brief overview of hit models and target theory in Section 1. We give a detailed development of the tumour growth multinomial model in Section 2. In Section 3 we review existing classical TCP models. Based on the multinomial model, we propose a new expression of TCP in Section 3 and NTCP in Section 4. In Section 5, we integrate the cell proliferation into our approach. Numerical results are presented in Section 6. Finally, the main results, limits and perspectives of the multinomial model are drawn in Section 7.

\section{Radiation, hit models and target theory}

The main notations used thereafter are presented in Table 1.

\subsection{Hit process}

For a radiation dose $u_{0}$, the number of delivered radiation particles is

$$
n_{p}=u_{0} \rho
$$


Table 1

Main notations

\begin{tabular}{|c|c|}
\hline \multicolumn{2}{|c|}{ Notations } \\
\hline Not. & Definition \\
\hline$k$ & discrete time \\
\hline$n_{p}$ & number of delivered radiation particles per a radiation dose $u_{0}$ \\
\hline$q_{h}$ & probability that a radiation particle hits a target \\
\hline$N_{h}$ & number of radiation particles that hit a critical target \\
\hline$q_{c}$ & probability of cell death after radiation \\
\hline$p_{c}$ & survival probability of a cell after treatment \\
\hline$v$ & voxel (subvolume) of a $3-\mathrm{D}$ scan \\
\hline$Z_{k}$ & number of deactivated targets in the cell \\
\hline$\Pi$ & transition matrix associated with the Markov chain $\left(Z_{k}\right)$ \\
\hline $\mathbf{P}$ & matrix associated with treatment effects \\
\hline $\mathbf{R}$ & matrix associated with cell repair process \\
\hline$X_{k, v}(i)$ & number of cancer cells in state $i$ in the voxel $v$ at time $k$ \\
\hline $\bar{X}_{k, v}(i)$ & number of normal cells in state $i$ in the voxel $v$ at time $k$ \\
\hline$m$ & number of targets in a cancer cell \\
\hline$q$ & probability to deactivate a target in a cancer cell \\
\hline$r$ & probability for an inactived target to be reactivated in a cancer cell \\
\hline$n_{0}$ & initial total number of cancer cells in the tumour \\
\hline$n_{t}$ & total number of tumour voxels \\
\hline$n_{v}$ & initial number of cancer cells in a tumour voxel $v$ \\
\hline $\bar{m}$ & number of targets in a normal cell \\
\hline $\bar{q}$ & probability to deactivate a target in a normal cell \\
\hline $\bar{r}$ & probability for an inactive target to be reactivated in a normal cell \\
\hline $\bar{n}_{0}$ & initial total number of normal cells in the irradiated zone \\
\hline $\bar{n}_{t}$ & total number of normal tissue voxels \\
\hline $\bar{n}_{v}$ & initial number of cells in a normal tissue voxel $v$ \\
\hline $\bar{n}$ & the complication threshold number of dead normal cells \\
\hline
\end{tabular}


with $\rho$ the number of radiation particles per unit dose and the subscript $p$ corresponds to particules. Thereafter, $n_{p}$ is assumed to be an integer for simplification. The underlying assumptions ${ }^{23}$ of the hit models are:

(1) a cell has at least one critical target;

(2) the probability $q_{h}$ (the subscript $h$ corresponds to hit) that a radiation particle hits a critical target is the same for all targets;

(3) the hit events are independent from each other.

Let $N_{h}$ be the random variable denoting the number of radiation particles that hit a given target. Then, $N_{h} \sim \mathcal{B}\left(n_{p}, q_{h}\right)$ (binomial distribution) and the probability that exactly $j$ radiation particles hit a given critical target is:

$$
\operatorname{Pr}\left(N_{h}=j\right)=\left(\begin{array}{c}
n_{p} \\
j
\end{array}\right) q_{h}^{j}\left(1-q_{h}\right)^{n_{p}-j}, \quad j=0,1,2, \cdots, n_{p} .
$$

If $n_{p}$ is large enough and $q_{h}$ is low, such as $n_{p} q_{h}=\lambda \in[0,10]$, the distribution of $N_{h}$ can be approximated by the Poisson distribution $\mathcal{P}(\lambda)$. Fixing

$$
\alpha=\rho q_{h},
$$

then according to (1.1), $\lambda=n_{p} q_{h}=\alpha u_{0}$ and the probability that exactly $j$ radiation particles hit a target becomes

$$
\operatorname{Pr}\left(N_{h}=j\right)=\frac{\left(\alpha u_{0}\right)^{j}}{j !} e^{-\alpha u_{0}}, \quad j=0,1,2, \cdots
$$

$\alpha u_{0}$ is the expected number of primary lesions caused by the radiation dose $u_{0}$ and the parameter $\alpha$ can be interpreted as a characteristic of the damage process itself, i.e. radiosensitivity in its literal sense ${ }^{28}$. There are several classes of hit models classified by the numbers of targets and hits. 


\subsection{Single target-single-hit models}

In single target-single-hit models, it is assumed that a cell has one lethal target and that the cell dies when its target is hit by one or more radiation particles. Therefore, the cell dies with probability

$$
q_{c}:=P\left(N_{h} \geq 1\right)=1-e^{-\alpha u_{0}} .
$$

\subsection{Single target-multi-hit models}

In single target-multi-hit models, the cell dies when the target is hit by at least $h_{t}$ radiation particles, where $h_{t}$ is a threshold number of cell inactivations also called extrapolation number. Therefore, the probability that the cell dies is

$$
q_{c}:=P\left(N_{h} \geq h_{t}\right)=\sum_{j=h_{t}}^{\infty} \frac{\left(\alpha u_{0}\right)^{j}}{j !} e^{-\alpha u_{0}}=1-e^{-\alpha u_{0}}-\cdots-\frac{\left(\alpha u_{0}\right)^{h_{t}-1}}{\left(h_{t}-1\right) !} e^{-\alpha u_{0}} .
$$

\subsection{Linear-quadratic model}

The linear-quadratic model considers that a cell contains one single target.

This model is based on a radiobiological approach ${ }^{1}$ : after a radiation dose $u_{0}$, the cell dies either by a single lethal event on the target which is represented by a linear component or by the combination of two sublethal events close enough in time and space, which is represented by a quadratic component. 
Thus, the cell death probability after a radiation dose $u_{0}$ is defined as

$$
q_{c}=1-p_{c}=1-e^{-\left(\alpha u_{0}+\beta u_{0}^{2}\right)},
$$

where $\alpha$ and $\beta$ are two positive parameters.

Dawson and Hillen ${ }^{4}$ provide a detailed explanation using radiation physics and leading to (1.7). Note that the linear quadratic (LQ) model has been gaining popularity these recent years ${ }^{2}$.

However, under the single-target assumption, the surviving cell population is homogeneous in terms of radiation injuries in cells. That is why we revisite in this paper the target modeling theory in a stochastic framework able to handle the heterogeneity of damages.

\subsection{Multi-target-single-hit models}

Here, the cell has $m$ distinct targets and dies when the $m$ targets have been inactivated. Each target can be deactivated by a single hit. According to (1.5), this event occurs with probability $1-e^{-\alpha u_{0}}$. Under the assumption that the deactivation of targets are independent events, the probability that the cell dies is

$$
q_{c}=\left(1-e^{-\alpha u_{0}}\right)^{m}
$$

Similarly to the single target models, the variation in cell radio-sensitivity has not been taken into account and all cells have the same survival probability $p_{c}=1-q_{c}$ 


\section{Multinomial model of a tumor}

Two main aspects of tumor growth are generally missing in the typical hit models: (i) the heterogeneity of damages induced by radiations in the cancer cell population after each dose fraction and (ii) the target reparation between two consecutive dose fractions. Thereafter, we thus propose to take these two issues into account in the multinomial model ${ }^{29}$. In this section, we detail the multinomial model without the cell proliferation which will be introduced afterward in Section 5.

\subsection{Radiation scheduling}

We restrict our study to fractionated radiation schedules that have 5 dose fractions per week. More specifically, the first fraction is given on Monday morning, and there is no treatment on the weekends. The treatment is based on a static (i.e. fraction sizes do not vary over time) scheme illustrated in Figure 1 by an impulse train $u(k)$ in which $u_{0}$ denotes the magnitude of each dose fraction (typically $u_{0}=2 G y$ ) and $k$ is the discrete time based on a daily

sampling rate. Thus, $d(k)=\sum_{i=0}^{k} u(i)$ is the cumulated dose of radiation up to time $k$. The treatment planning process is composed of the following steps:

- the patient image data is acquired, typically fully 3-D Computed Tomography (CT) scans;

- based on this CT scan, the physician outlines the tumor and important normal structures on a computer like the gross target volume, the clinical target volume, and the planning target volume;

- each 3-D CT scan is decomposed into subvolumes called voxels. It is sup- 
posed that each voxel $v$ received a uniform dose $u_{0, v}$ per fraction, but the latter may vary from a voxel to another one.

\subsection{Heterogeneity of cell states after radiations}

We consider that a cell contains $m$ targets. Each target can be made inactive by radiation particules and the cell death occurs when the $m$ targets are deactivated. After a fraction of treatment, a cell may have $m+1$ possible states:

- state $i$, the cell has $i$ inactive targets, $i \in\{0,1, \ldots, m-1\}$, these are the $m$ states of a surviving cell;

- state $m$, the cell having $m$ inactive targets is a dead cell.

Figure 2 shows the case of a 3 -target cell and the corresponding cell states.

\subsection{Discrete-time Markov chain model of a cancer cell}

A discrete-time Markov chain model is proposed to describe the heterogeneity of cellular damages during the fractionated treatment.

Let us first consider a single cell composed of $m$ targets. Let $Z_{k}$ be the random variable describing the state of the cell at time $k, Z_{k}=i \in\{0,1, \cdots, m\}$ is the number of deactivated targets at time $k$. We suppose that $\left(Z_{k}\right)$ is a discretetime Markov chain, i.e. the cell state at time $k+1$ only depends on the current state at time $k$ and let $\Pi$ the corresponding transition matrix. We determine thereafter the expression of $\Pi$ which models both of effects of dose fractions and repair mechanisms. 


\subsubsection{Treatment effect modeling.}

We adopt the convention that the first row and first column of a matrix will be noted by the index value 0 . Denote $\mathbf{P}(i, j)$ the probability to deactivate $j$ targets at time $k+1$ when $i$ targets are disabled at time $k$. Let $q$ the probability to inactivate a target after a dose fraction $u_{0}$. Moreover, we suppose that the disabling of targets in the cell are independent events. Thus after applying a fraction dose, the possible states at time $k+1$ of a cell in state $i$ at time $k$ are $\{i, i+1, \ldots, m\}$. The cell may switch at time $k+1$ to the state $j$ by the deactivation of $j-i$ target(s) among the $m-i$ active ones. Consequently,

$$
\mathbf{P}(i, j)=\left(\begin{array}{c}
m-i \\
j-i
\end{array}\right) q^{j-i}(1-q)^{m-j}
$$

Then, we obtain the general expression of the matrix $\mathbf{P}$,

$$
\mathbf{P}(i, j)= \begin{cases}\left(\begin{array}{l}
m-i \\
j-i
\end{array}\right) q^{j-i}(1-q)^{m-j} & i \leq j \\
0 & j<i\end{cases}
$$

and the explicit expression is

$$
\mathbf{P}=\left(\begin{array}{cccc}
(1-q)^{m} & \left.\begin{array}{c}
m \\
1
\end{array}\right) q(1-q)^{m-1} & \ldots & q^{m} \\
0 & (1-q)^{m-1} & \ldots & q^{m-1} \\
\vdots & \vdots & \vdots & \\
0 & 0 & \ldots & q \\
0 & 0 & \ldots & 1
\end{array}\right) .
$$


Figure 3 shows the transition graph of the radiation process before taking the repair of inactive targets into account, for the case of a 3-target cell, i.e. $m=3$.

The target deactivation parameter $q$ is the probability to deactivate an active target after a radiation dose fraction $u_{0}$. To connect this parameter with the real treatment schedule, we consider the following possibilities:

- If we assume that a target is deactivated when it is hit by one or more radiation particules, then according to (1.4), we have

$$
q=P\left(N_{h} \geq 1\right)=1-e^{-\alpha u_{0}},
$$

where $N_{h}$ is the random number of radiation particules that hit a given target.

- According to the (LQ) model, recall that after a dose fraction $u_{0}$ a cell dies with probability $q_{c}=1-e^{-\alpha u_{0}-\beta u_{0}^{2}}$. However, it is clear that in our model $q_{c}=\mathbf{P}(0, m)=q^{m}$. Consequently,

$$
q=\left(1-e^{-\alpha u_{0}-\beta u_{0}^{2}}\right)^{\frac{1}{m}}
$$

\subsubsection{Reparation modeling in surviving cells.}

We introduce now repair mechanisms of deactivated targets which occur between the application of two consecutive dose fractions. Let $r$ the probability of an inactive target in a living cell to revive during the period that separates two consecutive dose fractions. We assume that any target can be repaired independently from each other. The possible states at time $k+1$ of a cell in state $i$ at time $k$ are $\{0,1, \ldots, i\}$. Denote $\mathbf{R}(i, j)$ the probability that the cell 
switches at time $k+1$ to the state $j$. Since $i-j$ targets among the $i$ inactive targets are repaired, then

$$
\mathbf{R}(i, j)= \begin{cases}\left(\begin{array}{l}
i \\
j
\end{array}\right) r^{i-j}(1-r)^{j} & j \leq i<m \\
0 & i<j .\end{cases}
$$

For $i=m, \mathbf{R}(m, m)=1$ and $\mathbf{R}(m, j)=0$ for all $j \neq m$. The explicit expression is

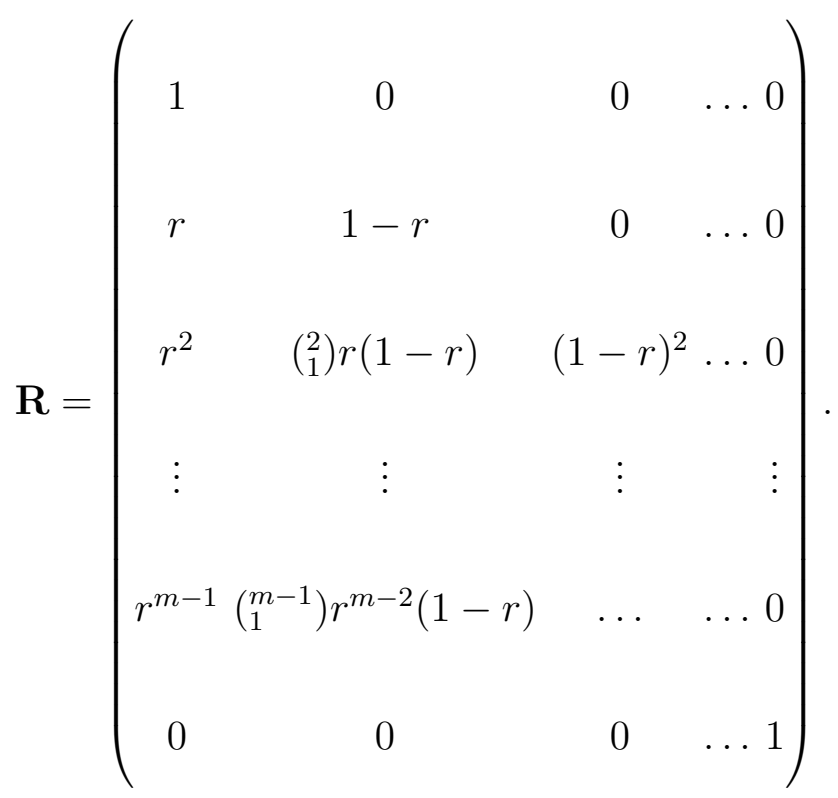

\subsubsection{Transition matrix of $\left(Z_{k}\right)$.}

We model the dynamics of the Markov chain $\left(Z_{k}\right)_{k \in \mathbf{N}}$ by taking firstly the effects of dose fractions and secondly repair mechanisms into account as follows

$$
\Pi=\mathbf{P R}
$$


where $\mathbf{P}$ models the effects of dose fractions, and $\mathbf{R}$ describes repair mechanisms.

In the case of a 3-target cell,

$$
\Pi=\left(\begin{array}{cccc}
\left(r q+q^{\prime}\right)^{3}-(r q)^{3} & 3 r^{\prime} q q^{2}+6 r r^{\prime} q^{2} q^{\prime} & 3 q^{2} q^{\prime} r^{\prime 2} & q^{3} \\
r q^{\prime 2}+2 r^{2} q q^{\prime} & r^{\prime} q^{\prime 2}+4 r r^{\prime} q q^{\prime} & 2 r^{\prime 2} q q^{\prime} & q^{2} \\
r^{2} q^{\prime} & 2 r r^{\prime} q^{\prime} & r^{\prime 2} q^{\prime} & q \\
0 & 0 & 0 & 1
\end{array}\right)
$$

where $q^{\prime}=1-q$ and $r^{\prime}=1-r$. Figure 4 shows the transition graph corresponding to the Markov chain $\left(Z_{k}\right)$ after taking the repair of inactive targets into account.

\subsubsection{Probability distribution of $Z_{k}$.}

Set $\nu_{k}=\left(\nu_{k}^{0}, \ldots, \nu_{k}^{m}\right)$ the probability distribution vector of $Z_{k}: \nu_{k}^{i}=P\left(Z_{k}=\right.$ $i)$. Using the Markov chain property, $\nu_{k}$ may be expressed in terms of $\Pi$ and $\nu_{0}$ :

$$
\nu_{k}=\nu_{0} \Pi^{k}
$$

If we assume that the cell is initially in state 0 , then $\nu_{0}=(1,0, \ldots, 0)$ and

$$
\nu_{k}^{i}=\Pi^{k}(0, i) \quad i \in\{0, \ldots, m\} .
$$




\subsubsection{Comparison with hit models.}

The multi-target-single-hit model is a special case of the multinomial model by considering that all sublethal damages are completely repaired between dose fractions, i.e. by taking the repair parameter $r$ equal to 1 . Indeed, when $r=1$, a cell is either dead or in state 0 , then the surviving cell population is homogeneous. This and assuming $q$ verifies (2.4) lead to the case of the multi-target-single-hit model.

Similarly, if we fix the target number parameter $m=1$ and the deactivation target parameter $q$ equals to (2.5), we get the the linear quadratic model.

\subsection{Multinomial model of the tumour}

Consider a group of $n_{0}$ cells that compose the tumour. We divide the tumour into $n_{t}$ voxels. We suppose that a voxel $v$ of the computerized tomography scan contains $n_{v}$ cells i.e.

$$
n_{0}=\sum_{v=1}^{n_{t}} n_{v}
$$

In each voxel $v$ we assume that:

- cells behave independently and with the same dynamics (cf Section 2.3);

- the probability $q_{v}$ to deactivate a target in a cell is the same for all cells located in $v$;

- the probability of target repair $r$ is constant and does not depend on $v$.

For each cell $j$, we associate a discrete-time Markov chain $\left(Z_{k}^{(j)}\right)$, where $Z_{k}^{(j)}$ is the random number of deactivated targets. Therefore, the Markov chains $\left\{\left(Z_{k}^{(j)}\right)\right\}_{j \in\left\{1, \ldots, n_{v}\right\}}$ are independent and have the same transition matrix $\Pi_{v}$. 
Let $X_{k, v}(i)$ be the random number of cells in state $i \in\{0, \ldots, m\}$, at time $k$, among the $n_{v}$ initial cells in the voxel $v$. Since $Z_{k}^{(1)}, \ldots, Z_{k}^{\left(n_{v}\right)}$ are i.i.d., and follow a categorial distribution, then $\left(X_{k, v}(0), \cdots, X_{k, v}(m)\right)$ follows a multinomial distribution with parameters $n_{v}$ and $\nu_{k}=\left(\nu_{k}^{0}, \ldots, \nu_{k}^{m}\right)$. Consequently, $\operatorname{Pr}\left(X_{k, v}(0)=h_{0}, \ldots, X_{k, v}(m)=h_{m}\right)$, is given by

$$
\left\{\begin{array}{cc}
\frac{n_{v} !}{h_{0} ! \ldots h_{m} !}\left(\nu_{k}^{0}\right)^{h_{0}} \ldots\left(\nu_{k}^{m}\right)^{h_{m}} & \text { if } \sum_{i=0}^{m} h_{i}=n_{v} \\
0 & \text { otherwise }
\end{array}\right.
$$

where $h_{i}, i \in\{0, \ldots, m\}$, are non-negative integers.

Note that the number $X_{k, v}(i)$ of cells in state $i$ at time $k$ follows the binomial distribution:

$$
X_{k, v}(i) \sim \mathcal{B}\left(n_{v}, \Pi_{v}^{k}(0, i)\right)
$$

Consequently, its average number is

$$
E\left(X_{k, v}(i)\right)=n_{v} \Pi_{v}^{k}(0, i)
$$

\subsection{Number of surviving cells}

In the multinomial model, the number of surviving cells in the voxel $v$ after the $k^{t h}$ dose fraction is given by

$$
N_{k, v}=X_{k, v}(0)+\ldots+X_{k, v}(m-1)=n_{v}-X_{k, v}(m) .
$$


Since $X_{k, v}(m) \sim \mathcal{B}\left(n_{v}, \Pi_{v}^{k}(0, m)\right)$, then

$$
N_{k, v} \sim \mathcal{B}\left(n_{v}, 1-\Pi_{v}^{k}(0, m)\right)
$$

This implies that

$$
E\left(N_{k, v}\right)=n_{v}\left(1-\Pi_{v}^{k}(0, m)\right)
$$

and

$$
\operatorname{Var}\left(N_{k, v}\right)=n_{v}\left(1-\Pi_{v}^{k}(0, m)\right) \Pi_{v}^{k}(0, m)
$$

\section{Tumor Control Probability}

The probability that no cancer cell remains in a tumor after radiation is known as the Tumor Control Probability (TCP). This probability may be useful to evaluate either the quality of a treatment planning or for its optimization.

Recall that the tumor is decomposed into $n_{t}$ voxels (cf Section 2.4) and these voxels are supposed to be independent, then the TCP at $k$ is given by

$$
T C P_{k}=\prod_{v=1}^{n_{t}} V C P_{k, v}
$$

where $V C P_{k, v}$ denotes the Voxel Control Probability at time $k$, i.e. the probability of having no cancer cell in the voxel $v$.

Expressions of $V C P$

The binomial and Poisson formulations of $V C P$, for a uniformly irradiated volume $v$ (voxel), are generally used in radiotherapy planning ${ }^{30-32}$. First, let 
$p_{c, v}(k)$ denote the probability that a cancer cell located in the voxel $v$ survives after $k$ dose fractions with magnitude $u_{0}$. Since we have supposed that all cells behave independently, then the number of surviving cancer cells after the $k^{\text {th }}$ dose fraction follows a binomial distribution $\mathcal{B}\left(n_{v}, p_{c, v}(k)\right)$, and we obtain

$$
V C P_{k, v}=\left(1-p_{c, v}(k)\right)^{n_{v}} .
$$

When the distribution $\mathcal{B}\left(n_{v}, p_{c, v}(k)\right)$ may be approximated by the Poisson distribution $\mathcal{P}\left(n_{v} p_{c, v}(k)\right)$, we have

$$
V C P_{k, v}=e^{-n_{v} p_{c, v}(k)} .
$$

The main drawback of hit models presented in Section 1 is to ignore the repair mechanism and the heterogeneous distribution of the cellular damages over the tumor and over time, since $p_{c, v}(k)$ is defined as

$$
p_{c, v}(k)=\left(p_{c, v}\right)^{k}
$$

where $p_{c, v}=p_{c, v}(1)$ is determined by equations (1.5), (1.6), (1.7) and (1.8) according to each model. The above relation (3.4) means that after each dose fraction a surviving cell has the same probability $p_{c, v}$ to survive after the next dose fraction.

However, considering the multinomial model presented in Section 2.4, the cell radiation sensitivity increases with the number of dose fraction and for a cell initialy in state 0 we have

$$
p_{c, v}(k)=1-\Pi_{v}^{k}(0, m) .
$$


Then, the multinomial $V C P$ at time $k$ is defined according to (3.2) as

$$
V C P_{k, v}=\left(\Pi_{v}^{k}(0, m)\right)^{n_{v}}
$$

Therefore, according to (3.1) we obtain the TCP for the whole tumour :

$$
T C P_{k}=\prod_{v=1}^{n_{t}}\left(\Pi_{v}^{k}(0, m)\right)^{n_{v}}
$$

\section{Normal Tissue Complication Probability}

The main undesirable effect due to radiotherapy is the irradiation of normal tissue. Normal organs and tissues have different responses to radiations due to their distinct architecture. Niemierko and Goitein ${ }^{33}$ proposed a division of normal tissues into three different architectures: serial architecture (e.g. nerves or spinal cord), parallel architecture (e.g. kidney, liver or lung) and graduated response (e.g. skin or mucous membranes).

We restrict ourselves to the parallel architecture. It is supposed that organs are composed of functional subunits (FSUs) and that organ function is compromised when a certain critical fraction of these FSUs is damaged.

The Normal tissue Voxel Complication Probability, noted $N V C P_{k, v}$, is defined as the probability that a complication appears in a normal tissue voxel $v$ at time $k$. The Normal Tissue Complication Probability $N T C P_{k}$ in the whole tissue at time $k$ is the probability that at least one voxel among the $\bar{n}_{t}$ voxels of the normal tissue has been damaged. If we assume that radiation injury to 
voxels are independant events, then

$$
N T C P_{k}=1-\prod_{v=1}^{\bar{n}_{t}}\left(1-N V C P_{k, v}\right) .
$$

For a uniformly irradiated voxel with $\bar{n}_{v}$ FSUs and a reserve capacity of $(\bar{n}-1)$ FSUs, it is supposed that ${ }^{34,36}$ :

- a complication appear if the number of damaged FSUs exceeds $\bar{n}$;

- each FSU among the $\bar{n}_{v}$ ones is damaged with a probability $p_{F S U}$;

- all FSUs behave independently.

Let $N_{F S U}$ be the random number of damaged FSUs among the $\bar{n}_{v}$ ones after irradiation, then it has a Binomial distribution $\mathcal{B}\left(\bar{n}_{v}, p_{F S U}\right)$ and the probability of complication in the voxel $v$ can be expressed mathematically as

$$
N V C P_{v}=P\left(N_{F S U} \geq \bar{n}\right)=\sum_{i=\bar{n}}^{\bar{n}_{v}}\left(\begin{array}{l}
\bar{n}_{v} \\
i
\end{array}\right)\left(p_{F S U}\right)^{i}\left(1-p_{F S U}\right)^{\bar{n}_{v}-i}
$$

\section{Multinomial NVCP}

In our model, we suppose that each FSU is one normal cell. Assume that the dynamic of normal cells is similar to the one of cancer cells and given by the multinomial model, defined in Section 2.4, but with different values of the parameters. Then, a normal cell contains $\bar{m}$ targets, each of them is deactivated with a probability $\bar{q}$ and it is repaired with a probability $\bar{r}$. However, if the treatment plan is correctly designed, radiation doses are lower in the voxels of normal tissue than in the ones of the tumour. As a consequence, the probability 
that a radiation particle inactivates a target in a normal cell, is lower than for a cancer cell $(\bar{q}<q)$. Moreover, the repair mechanism for normal tissue cells is better for fractionated radiation than tumour cells for reasons which are not yet explained. Consequently, the probability for an inactive target to be reactivated is greater for a normal cell than for a cancer cell $(\bar{r}>r)$.

For simplicity, we use the same notation, as for cancer cells, to denote the probability $\bar{\Pi}_{v}^{k}(0, \bar{m})$ that a normal cell, located in a voxel $v$, is in state $\bar{m}$ at time $k$ (dead cell). $\bar{X}_{k, v}(\bar{m})$ is the number of dead cells at time $k$ among the $\bar{n}_{v}$ initial cells in a normal voxel $v$. Obviously, $\bar{X}_{k, v}(\bar{m})$ is the analogue of $X_{k, v}(m)$ (cf Section 2.4). Since the complication in the normal voxel $v$ occurs when the dead cell number is larger than $\bar{n}$, then the $N V C P_{v}$ expression at time $k$ can be written as

$$
N V C P_{k, v}=P\left(\bar{X}_{k, v}(\bar{m}) \geq \bar{n}\right)=\sum_{i=\bar{n}}^{\bar{n}_{v}}\left(\begin{array}{l}
\bar{n}_{v} \\
i
\end{array}\right)\left(\bar{\Pi}_{v}^{k}(0, \bar{m})\right)^{i}\left(1-\bar{\Pi}_{v}^{k}(0, \bar{m})\right)^{\bar{n}_{v}-i}
$$

Note that, equation (4.3) is a special case of (4.2) for $p_{F S U}=\bar{\Pi}_{v}^{k}(0, \bar{m})$. In particular, if complications only occur when all cells are killed i.e. $\bar{n}_{v}=\bar{n}$, the the definitions of NVCP and VCP coincide and the normal voxel complication probability becomes

$$
N V C P_{k, v}=\left(\bar{\Pi}_{v}^{k}(0, \bar{m})\right)^{\bar{n}_{v}} .
$$

Finally, integrating (4.3) in (4.1), we obtain the NTCP for the normal tissue in the irradiated zone. 


\section{$5 \quad$ Cell proliferation modeling}

First let us describe the new behavior of a given cell taking the reproduction phase into account. Once the $k^{\text {th }}$ dose fraction is applied, the cell may be damaged, thus repair mechanisms and proliferation follow until the $k+1^{\text {th }}$ dose fraction. The damage and repair phases are again modelled as in Section 2.3. We suppose that the cell may reproduce if only it remains in state 0 after the application of a dose fraction, which occurs with a probability $\mathbf{P}(0,0)=$ $(1-q)^{m}$. A cell that stays in state 0 , divides and gives birth to two daughter cells in state 0 with probability $\mu$ or it remains unchanged with probability $1-\mu$. It is convenient for our computation, in the case of cell division, to consider the mother cell is still alive and a new one (in state 0) is artificially added.

Consider a voxel $v$ that contains $n_{v}$ cells in state 0 . All cells behave as described previously and independently. Let $S_{1}$ be the random number of the additional cells in state 0 resulting from cell reproduction after applying the first dose fraction on the $n_{v}$ cells. Since a new cell is produced with a probability $\mu(1-$ $\left.q_{v}\right)^{m}$, then

$$
S_{1} \sim \mathcal{B}\left(n_{v}, \mu\left(1-q_{v}\right)^{m}\right)
$$

Consequently,

$$
\eta_{1}:=E\left(S_{1}\right)=n_{v} \mu\left(1-q_{v}\right)^{m} .
$$

The number $X_{1, v}^{p}(0)$ of cells in state 0 , at time $k=1$, after including the cell proliferation becomes

$$
X_{1, v}^{p}(0)=X_{1, v}(0)+S_{1},
$$

where $X_{1, v}(0)$ is the number of cells in state 0 after the first dose fraction and 
the repair phase, among the $n_{v}$ initial cells,

$$
X_{1, v}(0) \sim \mathcal{B}\left(n_{v}, \Pi_{v}(0,0)\right) .
$$

We replace $S_{1}$ in the next steps by its mean value $\eta_{1}=E\left(S_{1}\right)$ and recall that these new cells are in state 0 . We deduce that modeling the treatment effects, repair mechanisms and cell proliferation in the initial cell population of $n_{v}$ cells is considered to be equivalent to model the treatment effects and repair mechanisms on these cells and to add $\eta_{1}$ cells in state 0 . This analysis can be repeated at each time $k$ adding a number $\eta_{k}$ of cells in state 0 resulting from cell reproduction. The sequence $\eta_{k}$ is given recursively by

$$
\left\{\begin{array}{cc}
\eta_{0}= & n_{v} \\
\eta_{k+1}=\mu\left(1-q_{v}\right)^{m} \sum_{j=0}^{k} \Pi_{v}^{k-j}(0,0) \eta_{j} k \geq 0
\end{array}\right.
$$

and the new expression of the VCP becomes

$$
V C P_{k+1}=\prod_{j=0}^{k}\left(\Pi_{v}^{k+1-j}(0, m)\right)^{\eta_{j}} \quad k \geq 0 .
$$

\section{$6 \quad$ Numerical analysis}

\subsection{Comparative analysis with typical VCP/NVCP}

Recall that we have observed that classical hit models are particular cases of the multinomial model by fixing the target reparation parameter $r$ and $\bar{r}$ for 
cancer and normal cells respectively at $r=\bar{r}=1$ or by taking the single-target assumtion i.e. $m=1$. Therefore the comparison between VCP/NVCP typical model vs $\mathrm{VCP} / \mathrm{NVCP}$ multinomial model reduces to study the influence of the parameter $r$ (and $\bar{r}$ ) on the $\mathrm{VCP} / \mathrm{NVCP}$ models.

For simplicity we only consider one voxel. The multinomial VCP and NVCP equations (3.6) and (4.3) were implemented in the Matlab environment. The parameters were fixed to $m=3, q_{v}=0.7, n_{v}=10000$ for cancer cells; $\bar{m}=3$, $\bar{q}_{v}=0.6$ and $\bar{n}=\bar{n}_{v}=1000$ for normal cells. We chose parameter values avoiding numerical complications so that they permit to obtain numerical results without any specific biological interpretations. The values of the repair parameters for the multinomial $\mathrm{VCP} / \mathrm{NVCP}$ curves are $r=0.3$ and $\bar{r}=$ 0.5 ; and recall that $r=\bar{r}=1$ for the classical $\mathrm{VCP} / \mathrm{NVCP}$ models. The radiation treatment is decomposed into 50 dose fractions. Note that $V C P_{k, v}$ and $N V C P_{k, v}$ given by (3.6) and (4.3) respectively, are functions of $k$. In practice, it is more convenient to express them in terms of the cumulated dose $d(k)=k u_{0}$. Figure 5 presents the VCP/NVCP curves obtained as functions of the cumulated dose $d$. Blue lines denote the VCP curves; the solid and dashed lines correspond to $r=0.3$ and $r=1$ (typical assumption) respectively. Similary, red curves represent the NVCP with $\bar{r}=0.5$ (solid line) and $\bar{r}=1$ (dashed line). For each couple of VCP/NVCP curves, an optimal dose OD is estimated by maximizing the total dose with the constraint NVCP $\leq 0.05$.

The optimal total doses OD1 and OD2 are deduced from the NVCP curves, as

$$
\begin{gathered}
O D 1=\max \{d, \text { multinomial } N V C P(d) \leq 0.05\}, \\
O D 2=\max \{d, \text { typical } N V C P(d) \leq 0.05\} .
\end{gathered}
$$


We observe that the optimal dose OD2 $\approx 46 G y$ derived from the typical $\mathrm{VCP} / \mathrm{NVCP}$ curves $(r=\bar{r}=1)$ is clearly greater than the optimal dose OD1 $\approx 27 G y$ corresponding to the multinomial VCP/NVCP curves calculated with $r=0.3$ and $\bar{r}=0.5$.

The above numerical results show an important difference between the outcome of each couple of the VCP/NVCP curves. This is mainly due to heterogeneity of cell states and reparation process in cancer and normal cells, quantified by the multinomial modeling. As a therapeutical consequence, this approach may reduce the total radiation dose to be delivered and thus the risk of side effects.

\subsection{VCP calculation for a clinical schedule}

We present here a VCP calculation taking into account the proliferation of cells with a given real value of dose fraction $u_{0}$. The VCP equation (5.5) was implemented in the Matlab environment using for the parameter $q$ the relation (2.5) depending on $\alpha$ and $\beta$ (1.7). The parameters were fixed to $m=3, r=0.3$, $\mu=1, n_{v}=10000$ and we consider the couple $(\alpha, \beta)=(0.3,0.03)$ used in $^{35}$.

Figure 6 displays the variation of the VCP as function of the cumulated dose $d$. The solid curve represents the VCP function for $u_{0}=1 G y$ and the dashed one corresponds to $u_{0}=2 G y$. This shows the sensitivity of the VCP prediction to the $u_{0}$ values. It is expected that the efficiency of the treatment increases with the value of the dose fraction $u_{0}$. 


\section{Conclusion}

In this paper, we proposed a multinomial model suited to treatment in radiotherapy, which takes into account (i) the variety of cell responses according to their biological states and (ii) the repair mechanisms that occur between dose fractions. Moreover we included the proliferation of cells that may produce during the treatment period. The radio-sensitivity of cells due to their positions in the cell cycle and the angiogenesis process (tumour blood supply) have not been considered in our model.

The new model is composed of only three parameters: the number $m$ of critical targets which allows to quantify the heterogeneity of intracellular damages during the treatment plan, the probability $q$ for a target to be deactivated by radiation and the probability $r$ for an inactive target in an alive cell to be reactivated. The parameter $q$ is related to the radiation dose $u_{0}$ through the intrinsic sensitivity of a target to radiation. Moreover, the multinomial model is a generalization of typical hit models. Based on the multinomial model, new expressions of the TCP and NTCP have been proposed for nonuniform radiations which permits to deduce the optimal total dose to be delivered. We point out the important influence of the repair parameter $r$ which could lead to reduce both the total radiation dose to be delivered and the risk of side effects.

\section{References}

1 Brener DJ, Hall EJ. The origin and basis of the linear quadratic model. Int J Radiat Oncol Biol Phys 1992, 23:252. 
2 Arun Chougule, Supe SJ. Early skin reactions in head and neck malignancy treated by twice-daily fractionated radiotherapy-estimation of alpha/beta of LQ model 1993, 39 (9):1335-1342.

3 Zaider M, Minerbo GN. Tumour control probability: a formulation applicable to any temporal protocol of dose delivery. Phys. Med. Biol. 2000, 45:279-293.

4 Dawson A, Hillen T. Derivation of the tumour control probability (TCP) from a cell cycle model. Computational and Mathematical Methods in Medicine 2006, 7:121-141.

5 Gay HA, Niemierko A. A free program for calculating EUD-based NTCP and TCP in external beam radiotherapy. Physica Medica 2007, 23:115-125.

6 Holthusen H. Erfahrungen über die Verträglichkeitsgrenze für Röntgenstrahlen und deren Nutzanwendung zur Verhütung von Schäden. Strahlentherapie 1936, 57:254-269.

7 Kim Y, Tomé WA. On the impact of functional imaging accuracy on selective boosting IMRT. Physica Medica 2009, 25:12-24.

8 Suntharaligam N, Padgorsak EB, Hendry JH. Basic Radiobiology. In Radiation Oncology Physics. Edited by Padgorsak EB, Vienna, Austria: International Atomic Energy Agency 2005:485-504.

9 Fowler J. The Linear-quadratic Formula and Progress in Fractionated Radiotherapy. Br. J Radiol 1989, 62:679-694.

10 Sachs RK, Hlatky LR, Hahnfeldt P. Simple ODE Models of Tumor Growth and Anti-Angiogenic or Radiation Treatment. Mathematical and Computer Modelling 2001, 33:1297-1305.

11 Hillen T, de Vries G, Gong J, Finlay C. From cell population models to tumor control probability: including cell cycle effects. In Acta Oncologica 2010:1-9. 
12 Kirkby NF, G BN, Faraday DBF. Mathematical modelling of the response of tumour cells to radiotherapy. Nuclear Instruments and Methods in Physics Research. Section B: Beam Interactions with Materials and Atoms 2002, 188:210-215.

13 Dessauer F. Uber einige Wirkungen von Strahlen. I. Z. Phys. 1922, 12:3847.

14 Blau M, k Altenburger. Uber einige Wirkungen von Strahlen. II. Z. Phys. 1922, 12:315-329.

15 Crowther JA. Some considerations relative to the action of X-rays on tissue cells. In Proceedings of the Royal Society of London: Series B 1924:207211.

16 Atwood KC, Norman A. On the interpretation of multi-hit survival curves. Proc. Natl. Acad. Sci. USA 1949, 35:696-709.

17 Lea DE. Actions of Radiations on Living Cells. Cambridge, UK: Cambridge University Press 1955.

18 Pollard EC, Guild WR, Hutchinson F, Setlow RB. The Direct Action of Ionizing Radiation on Enzymes and Antigen. Progress in Biophysics 1955, 5:72-108.

19 Pollard EC. Radiation Inactivation of Enzymes, Nucleic Acids and Phage Particles. Rev. Mod. Phys. 1959, 31:273-281.

20 Fowler JF. Differences in Survival Curve Shapes for Formal Multi-target and Multi-hit Models. Physics in Medicine and Biology 1964, 9:177-188.

21 Sy WNC, Han P. Analysis of a stochastic model of cell survival. Journal of Theoretical Biology 1982, 96:309-326.

22 Ditlov V. The evolution of track theory throughout the history of the international solid state detector conferences. International Conference on Nuclear Tracks in Solids 2001, 34:19-26. 
23 Satow T, Kawai H. Hit and Target Models for DNA Damage with Indirect Action. Computers and Mathematics with Applications 2006, 51:257-268.

24 Chapman JD. Target Theory Revisited: Why Physicists are Essential for Radiobiology Research. Clinical Oncology 2007, 19:S12.

25 Ditlov VA. Track theory and radiation effects. Radiation Measurements 2009, 44:1100-1104.

26 Dertinger H, Jung H Molecular Radiation Biology. New York: Springer Verlag 1970.

27 Datta R, Cole A, Robinson S. Use of track-end alpha particles from 241 Am to study radiosensitive sites in CHO cells. Radiat. Res. 1976, 65:139-151.

28 Hanin LG, Klebanov LB, Yakovlev AY. Randomized multihit models and their identification. J. Appl. Prob. 1996, 33:458-471.

29 Keinj R, Bastogne T, Vallois P. A multinomial model of tumour growth treated by radiotherapy. In Proceedings of the 11th IFAC Symposium on Computer Applications in Biotechnology; Leuven, Belgium 2010.

30 Murno TR, Gilbert CW. The relation between tumor lethal doses and the radiosensitivity of tumor cells. Br.J.Radiol. 1961, 34:283-287.

31 Brahme A. Dosimetric precision requirements in radiation therapy. Acta Radiol. Oncol. 1984, 23:379-391.

32 Brenner DJ. Dose, volume, and tumor-control predictions in radiotherapy. Int. J. Radiat. Oncol. Biol. Phys. 1993, 26:171-179.

33 Niemierko A, Goitein M. Calculation of normal tissue complication probability and dose-volume histogram reduction schemes for tissues with a critical element architecture. Radiother Oncol 1991, 20:166176.

34 A Jackson, GJ Kutcher, and ED Yorke. Probability of radiation-induced complications for normal-tissues with parallel architecture subject to nonuniform irradiation. Med. Phys., 20 (3):613-25, 1993. 
35 Brad Warkentin, Pavel Stavrev, Nadia Stavreva, Colin Field, B Gino Fallone. A TCP-NTCP estimation module using DVHs and known radiobiological models and parameter sets. Journal of Applied Clinical Medical Physics, 5 (1) 2004.

36 A Niemierko and M Goitein. Modeling of normal tissue-response to radiation-the critical volume model. Int. J. Radiat. Oncol. Biol. Phys., 25 (1):135-45, 1993. 


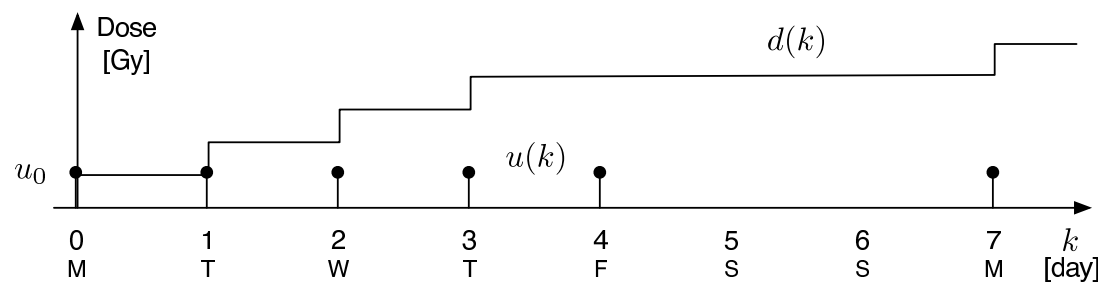

Figure 1. Radiation static scheme: $u(k)$ is an impulse train of dose fractions daily delivered, five days a week; $d(k)$ is the cumulated dose of radiation.
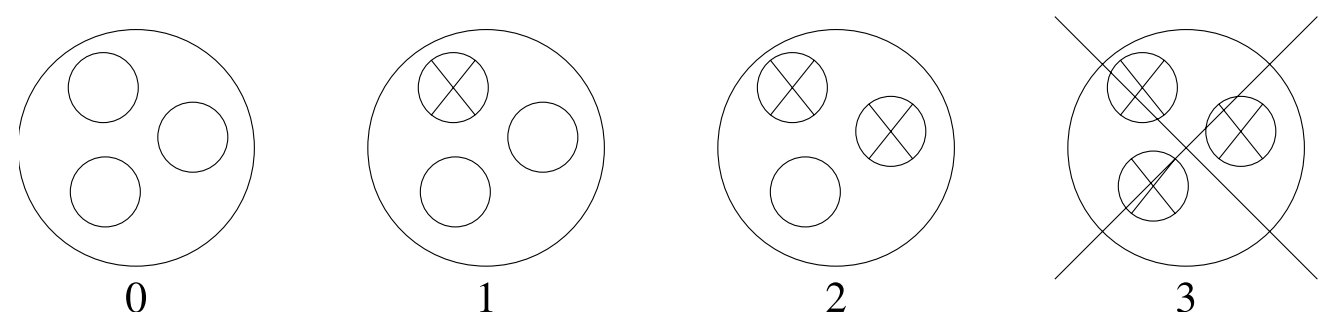

Figure 2. Heterogeneous states of a cell $(m=3)$ after radiation exposure. 


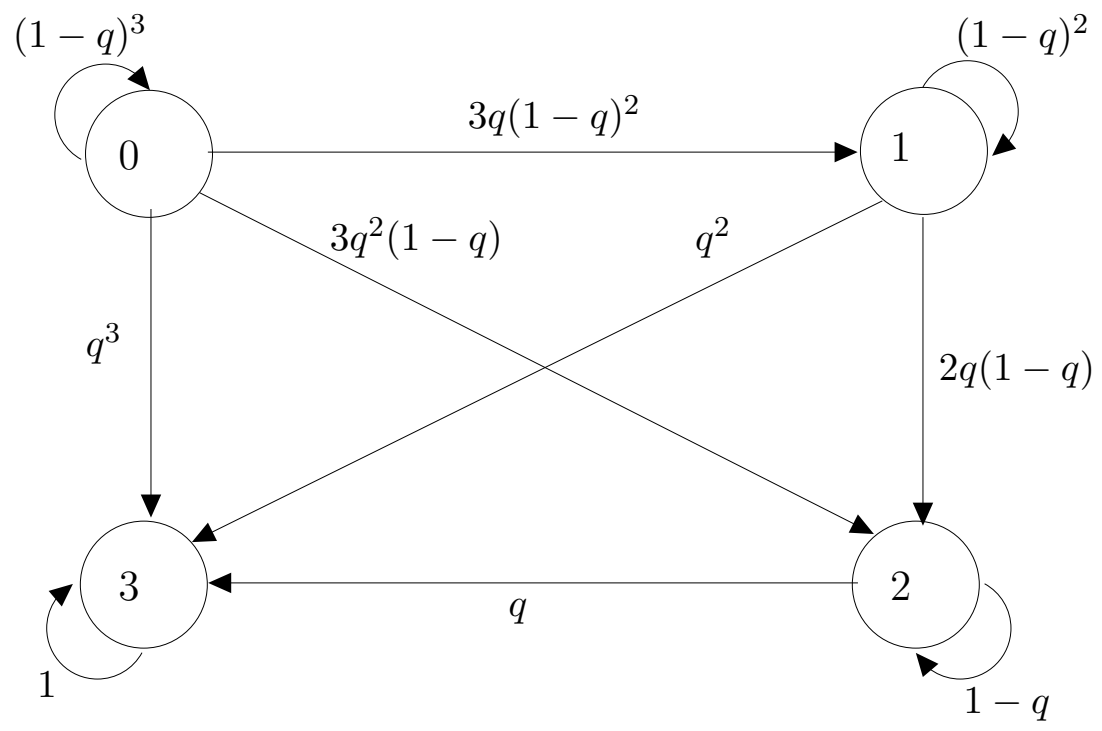

Figure 3. Transition graph of radiation process, with $m=3$ and before taking target repair mechanisms into account.

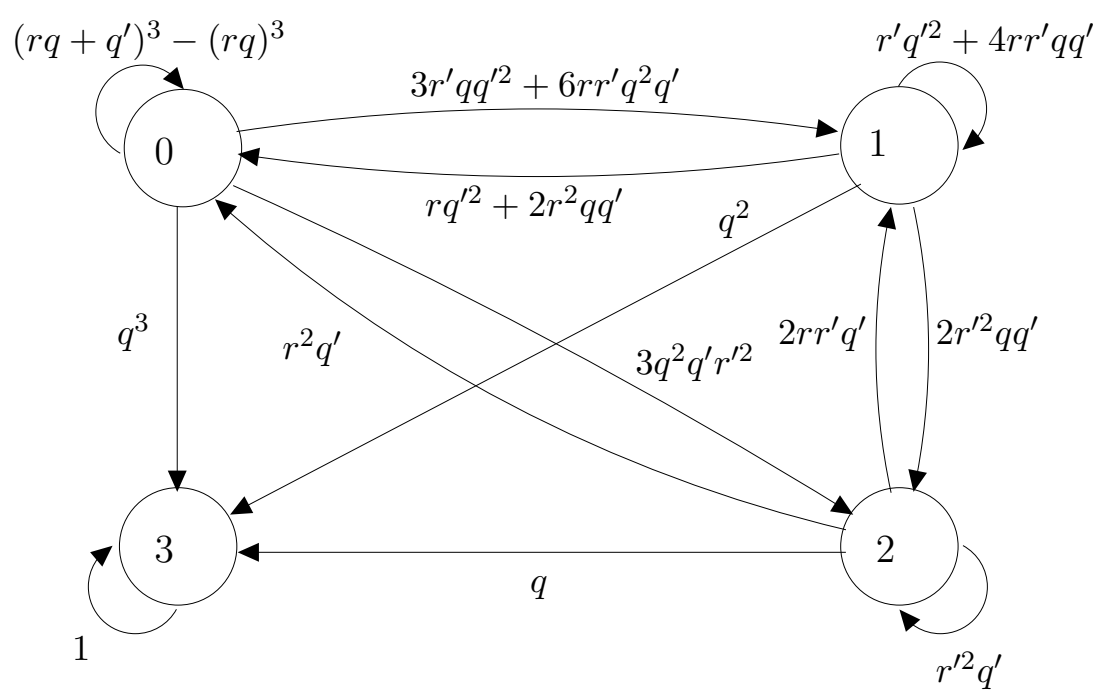

Figure 4. Transition graph of the Markov chain $\left(Z_{k}\right)_{k \in \mathbf{N}}$ where $m=3$ and after including the repair of inactive targets. 


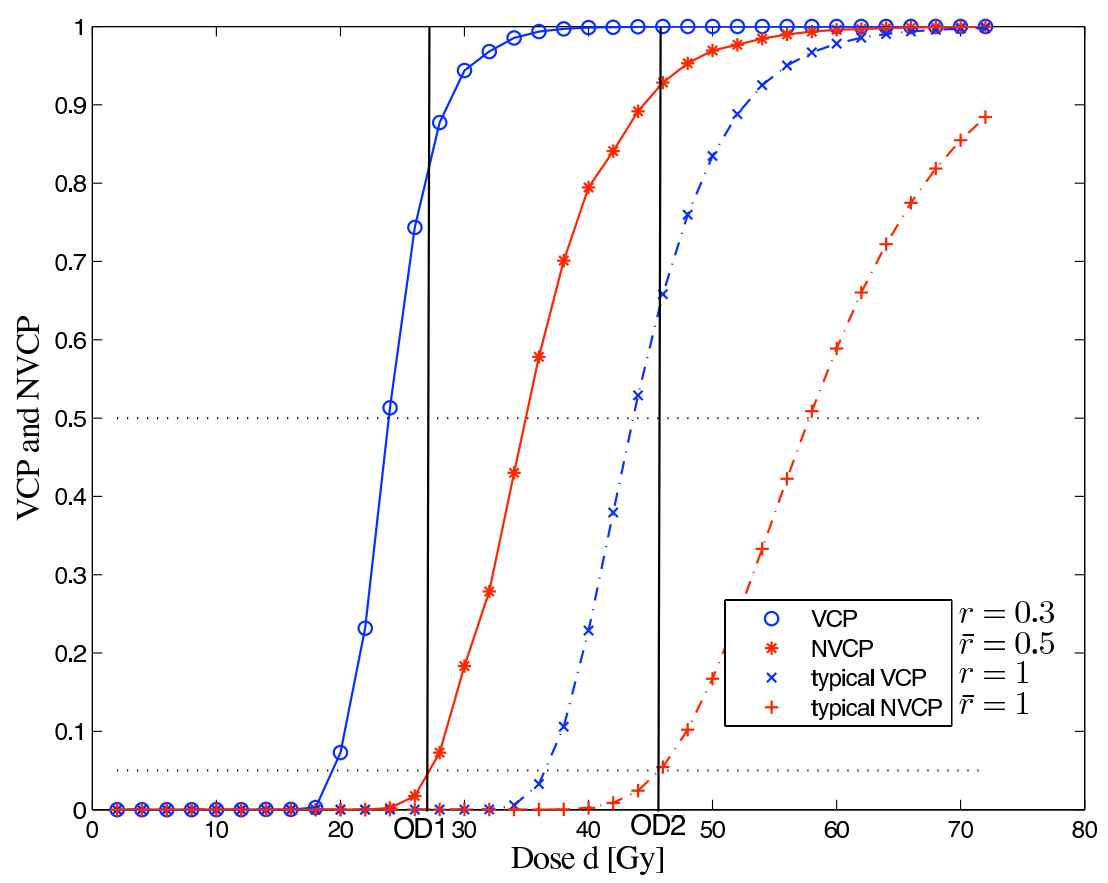

Figure 5. Calculated values of VCP and NVCP as functions of the cumulative dose $d$. Blue curves represent VCP functions: solid line for $r=0.3$ and the dashed line for $r=1$ (typical VCP). Red curves represent the NVCP functions, where $\bar{r}=0.5 \mathrm{in}$ solid line and $\bar{r}=1$ (typical NVCP) in dashed line. For $r=\bar{r}=1$, the corresponding optimal dose OD2, that maximizes the VCP with the constraint $N V C P \leq 0.05$, is larger then the optimal dose OD1 obtained with the other $\mathrm{VCP} / \mathrm{NVCP}$ functions. 


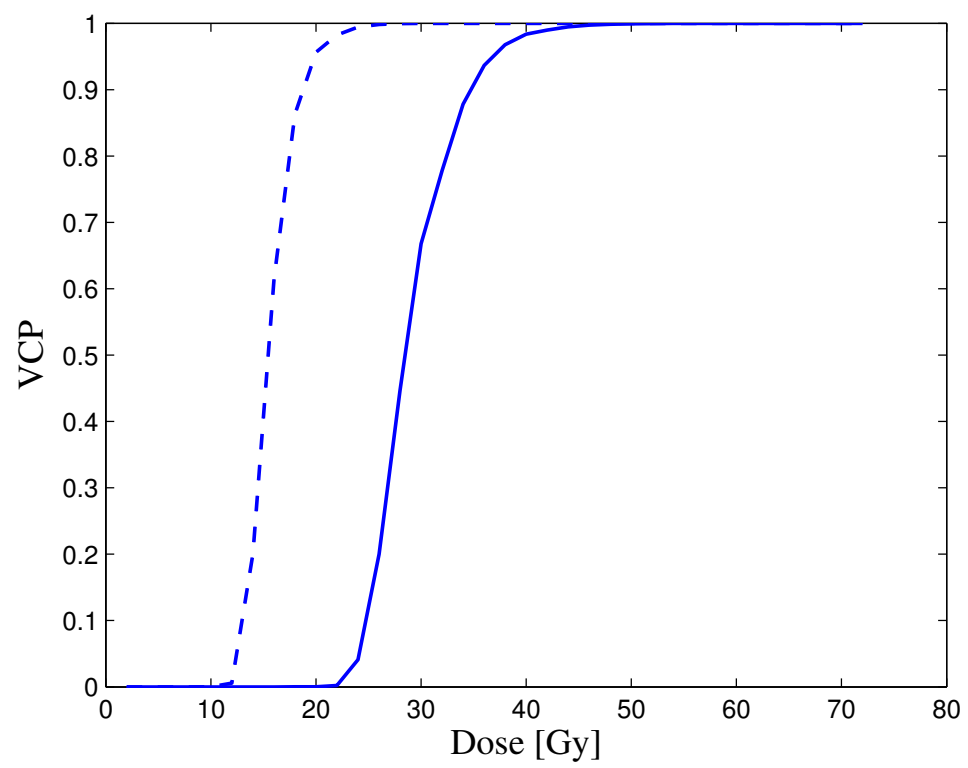

Figure 6. Calculated values of VCP as function of the cumulative dose $d$ taking into account the cell proliferation for the fixed parameters $m=3, r=0.3, \mu=1$, $n_{v}=10000,(\alpha, \beta)=(0.3,0.03)$. The solid curve represents the VCP function for $u_{0}=1 G y$ and the dashed one corresponds to $u_{0}=2 G y$. 